\title{
A note on determination of the diffuse-field sensitivity of microphones using the reciprocity technique
}

\section{Barrera Figueroa, Salvador; Jacobsen, Finn}

Published in:

Journal of the Acoustical Society of America

Link to article, DOI:

$10.1121 / 1.2950089$

Publication date:

2008

Document Version

Publisher's PDF, also known as Version of record

Link back to DTU Orbit

Citation (APA):

Barrera Figueroa, S., \& Jacobsen, F. (2008). A note on determination of the diffuse-field sensitivity of microphones using the reciprocity technique. Journal of the Acoustical Society of America, 124(3), 1505-1512. https://doi.org/10.1121/1.2950089

\section{General rights}

Copyright and moral rights for the publications made accessible in the public portal are retained by the authors and/or other copyright owners and it is a condition of accessing publications that users recognise and abide by the legal requirements associated with these rights.

- Users may download and print one copy of any publication from the public portal for the purpose of private study or research.

- You may not further distribute the material or use it for any profit-making activity or commercial gain

- You may freely distribute the URL identifying the publication in the public portal 


\title{
A note on determination of the diffuse-field sensitivity of microphones using the reciprocity technique ${ }^{\text {a) }}$
}

\author{
Salvador Barrera-Figueroa ${ }^{\text {b) }}$ and Knud Rasmussen ${ }^{\text {c) }}$ \\ Danish Primary Laboratory of Acoustics, Danish Fundamental Metrology, Matematiktorvet B307, \\ DK-2800 Lyngby, Denmark \\ Finn Jacobsen ${ }^{\text {d) }}$ \\ Acoustic Technology, Department of Electrical Engineering, Technical University of Denmark, Ørsteds \\ Plads B352, DK-2800 Lyngby, Denmark
}

(Received 12 December 2007; revised 29 May 2008; accepted 30 May 2008)

\begin{abstract}
The diffuse-field response of a microphone is usually obtained by adding a random-incidence correction to the pressure response of the microphone. However, the random-incidence correction is determined from a relative measurement, and its accuracy depends not only on the relative response at all angles of incidence but also on the accuracy of the frequency response at normal incidence. By contrast, this paper is concerned with determining the absolute diffuse-field response of a microphone using the reciprocity technique. To examine this possibility, a reciprocity calibration setup is used for measuring the electrical transfer impedance between a pair of microphones placed in a miniature $\left(2 \mathrm{~m}^{3}\right)$ reverberation room. The transfer function between the microphones is measured using fast Fourier transform analysis and pseudorandom noise. The calculation of the diffuse-field sensitivity involves (a) separation of the reverberant response from the total response, (b) determination of the reverberation time, and (c) averaging over space and frequency. The resulting diffuse-field correction is compared with an estimate of the random-incidence correction determined in an anechoic room and with a numerical prediction.
\end{abstract}

(C) 2008 Acoustical Society of America. [DOI: 10.1121/1.2950089]

PACS number(s): 43.38.Kb, 43.58.Vb [AJZ]

Pages: $1505-1512$

\section{INTRODUCTION}

The sensitivity of a microphone can be defined in terms of the sound field in which it is immersed. Uniform pressure conditions imply that the membrane of the microphone is exposed to a uniform sound pressure, for example, in a small coupler. Under free-field conditions, a plane wave impinges on the body of the microphone and the response of the microphone depends on the scattering caused by the rigid surfaces of the microphone and its radiation impedance. Finally, under ideal diffuse-field conditions, an infinite number of incoherent plane waves coming from random directions and having random phases and amplitudes impinges on the microphone; thus the diffuse-field sensitivity is the response of the microphone in a "perfectly diffuse sound field," an unrealizable, idealized concept that corresponds to an average over an infinite ensemble of realizations of sound fields composed of infinitely many plane waves with random phases arriving from all directions. ${ }^{1}$ Although such a sound field can be approximated in reverberation rooms, another quantity, the random-incidence sensitivity, is used conventionally as an equivalent to the diffuse-field sensitivity. The randomincidence sensitivity is the incoherent combination of the

\footnotetext{
"Portions of this work were presented in "An investigation of microphone calibration in a diffuse sound field," Proceedings of Inter-Noise 2007, Istanbul, Turkey, August 2007.

${ }^{b)}$ Electronic mail: sbf@dfm.dtu.dk

${ }^{c)}$ Electronic mail: kra@dfm.dtu.dk

${ }^{\mathrm{d})}$ Electronic mail: fja@elektro.dtu.dk
}

responses of the microphone at all possible directions. In practice this quantity is determined by averaging the response of the microphone to waves coming sequentially from all directions. ${ }^{2}$

Today practically all microphones are calibrated using pressure-field methods, although more than $90 \%$ of all microphones are used under diffuse-field or free-field conditions. The diffuse-field sensitivity of a microphone is in practice determined from its measured pressure sensitivity using empirical corrections that depend on the type and geometry of the microphone. ${ }^{3}$ The disadvantage of this method is that it gives rise to long delays when new transducers are introduced because it takes time for such corrections to be recognized internationally. Another problem is that some transducers, e.g., microelectromechanical system (MEMS) microphones, simply cannot be pressure calibrated.

The reciprocity technique for microphone calibration under pressure-field conditions in closed couplers was introduced in the $1940 \mathrm{~s},{ }^{4-7}$ and has since then been developed to a level of very high accuracy. The reciprocity technique yields the absolute sensitivity, and thus it is used in primary calibration. ${ }^{8}$ The free-field reciprocity technique was also introduced in the $1940 \mathrm{~s},{ }^{6,7}$ but it took much longer time to develop this method to a high level of accuracy, ${ }^{9}$ partly because of the extreme difference in signal levels (if the transmitter microphone is driven with $10 \mathrm{~V}$ the response from the receiver microphone will typically be less than $1 \mu \mathrm{V}$ ) and partly because anechoic rooms in practice are less than perfect combined with the fact that reflections have a serious 
influence. More recently, time-selective techniques based on advanced signal processing have been developed. ${ }^{10}$ Application of the time-selective techniques implies the possibility of doing free-field calibrations under nonanechoic conditions. Only a little has been published on diffuse-field calibration of microphones. ${ }^{11-13}$ The only fundamental studies were published about 40 years ago, ${ }^{11,12}$ and no standard for diffuse-field calibration of microphones has ever been developed, undoubtedly because diffuse-field reciprocity calibration of microphones is even more difficult than free-field reciprocity calibration.

The random-incidence sensitivity is conventionally determined from a pressure calibration combined with a random-incidence correction that is based on the free-field correction and an additional correction for nonaxial sound incidence, where the latter is obtained from relative measurements of the frequency response of the microphone in a free field when a succession of sound waves impinges on the microphone from all directions. ${ }^{2,14,15}$ Alternatively, it could be determined from a free-field calibration combined with the correction for nonaxial incidence. However, direct, absolute diffuse-field calibration would shorten the measurement chain. The purpose of this investigation is to establish a setup for reciprocity calibration of condenser microphones under diffuse-field conditions, to examine the sound field statistics, and to demonstrate that diffuse-field reciprocity calibration is possible with high precision. The investigated issues are (i) measurement of the transfer function between microphones in a reverberation room, (ii) separation of the direct and the reverberant responses using a time-selective technique, (iii) calculation of the diffuse-field sensitivity of laboratory standard (LS) microphones, (iv) averaging of the resulting sensitivity over frequency and space, and (v) validation of the diffuse-field response using a random-incidence estimate.

\section{THE DIFFUSE-FIELD SENSITIVITY}

Formally, the diffuse-field sensitivity is defined as follows: "for a sinusoidal signal of a given frequency in a diffuse sound field and for given environmental conditions, the quotient of the open-circuit voltage of the microphone by the sound pressure that would exist at the position of the acoustic center of the microphone in the absence of the microphone." ${ }^{\prime 6}$ However, a sinusoidal signal inevitably implies an interference field with large spatial variations of the sound pressure level, in obvious disagreement with the concept of a perfectly diffuse sound field. ${ }^{1,17-19}$ Diestel $^{11}$ and Nakajima $^{12}$ tacitly used a far more reasonable definition that, in fact, corresponds to the ensemble average of realizations of such interference fields, in other words, to the randomincidence sensitivity.

The electrical transfer impedance between two microphones in a diffuse field is ${ }^{11}$

$$
Z_{12}=\frac{u_{2}}{i_{1}}=M_{d, 1} \cdot M_{d, 2} \frac{1}{J_{d, 12}},
$$

where $u_{2}$ is the open-circuit voltage on the terminals of the receiver microphone, $i_{1}$ is the current through the terminals of the transmitter microphone, $M_{d, 1}$ and $M_{d, 2}$ are the diffuse- field sensitivities of microphones 1 and 2, respectively, and $J_{d, 12}$ is the reciprocity factor. The reciprocity factor, which follows from the relation between sound power emitted by the transmitter microphone and the resulting mean-square pressure in the reverberation room, ${ }^{11}$ is

$$
J_{d}=\left(\frac{6}{\pi \log e}\right)^{1 / 2}\left(\frac{1}{\rho_{0} f}\right)\left(\frac{V}{c T_{60}}\right)^{1 / 2},
$$

where $V$ is the volume of the reverberation room, $c$ is the speed of sound, $T_{60}$ is the Sabine reverberation time, $f$ is the frequency, and $\rho_{0}$ is the density of air. If a third microphone is coupled sequentially with microphones 1 and 2, then the absolute diffuse-field sensitivities of the three microphones can be determined by solving three equations that contain the three products of sensitivity. For instance, the diffuse-field sensitivity of microphone 1 can be obtained from

$$
M_{d, 1}^{2}=\frac{Z_{12} Z_{13}}{Z_{23}} \frac{J_{d, 12} J_{d, 13}}{J_{d, 23}} .
$$

Similar equations can be derived for microphones 2 and 3. A further simplification can be made if the reciprocity factor can be considered to be the same in the three measurements. Note, however, that the air absorption and thus the reverberation time, the speed of sound, and the density of air might change because of changes in the environmental conditions. Finally, the diffuse-field correction $C_{d}$ can be defined as the logarithmic ratio of the diffuse-field sensitivity to the pressure sensitivity,

$$
C_{d}=10 \log \left|\frac{M_{d}}{M_{p}}\right|^{2},
$$

where $M_{p}$ is the pressure sensitivity determined by reciprocity calibration (or from the electrostatic actuator response and the low-frequency sensitivity from a piston-phone calibration). This quantity will be compared with the randomincidence correction.

It should be added that the sound power emitted by a pure tone source in a reverberation room varies significantly with the position of the source and with the frequency at low frequencies, ${ }^{20,21}$ from which it follows that Eq. (2) is only valid on average: source position averaging is needed.

\section{REALIZATION OF A DIFFUSE SOUND FIELD}

It is a normal practice in pressure and free-field reciprocity calibration that the signals used are pure sinusoidal tones. This is particularly appropriate in free-field calibration because the signal-to-noise ratio is best using such a signal. In pressure calibration, signal levels are not the most important issue because the small cavities used normally give good signal-to-noise ratios. In principle, it is also possible to use pure tones in a reverberation room. The sound field in the room will be the product of a large number of interferences between reflected waves. This sound field has well defined statistical characteristics that make it possible to estimate, e.g., the spatial variation of the sound pressure in such a sound field. Above the Schroeder frequency, ${ }^{22}$ the meansquare pressure in a reverberant sound field generated by a pure tone, $\overline{p^{2}}$, may be regarded as a stochastic process which 
is a random function of the frequency ${ }^{22,23}$ and a random function of the position. ${ }^{18,19}$ Both functions have an exponential distribution. The normalized variance of the exponential distribution, that is, the ratio of the variance to the square of the mean, $\left.\sigma^{2} \overline{p^{2}}\right) / E^{2}\left(\overline{p^{2}}\right)$, equals 1 . This implies that the variations of the sound pressure with position and with frequency are very large.

The electrical transfer impedance is directly proportional to the sound pressure impinging on the receiver microphone. Thus, the resulting sensitivity will have similar statistical properties as the sound field generated by a pure tone source. On the other hand, if the reverberant response is to be separated from the total response, the length of the impulse response that corresponds to the measured transfer impedance as a function of the frequency must include the whole reverberant response. This requires a very fine sampling in the frequency domain that would make the application of sequential pure tone measurements prohibitively time consuming. A suitable solution is to use pseudorandom noise instead. Because of the fact that pseudorandom noise is a multitone signal, the resulting sound field will, at any frequency, have the same characteristics as the sound field generated by a pure tone. Therefore the use of pseudorandom noise reduces the measurement time dramatically. However, any signal processing technique capable of determining a sufficiently long impulse response will do; one might, for example, use frequency sweeps. ${ }^{24}$

\section{A. Spatial averaging}

In general, the variance of the average of a random variable, in this case the mean-square pressure (or the meansquare sensitivity) averaged over $n$ positions in the room, $\mathbf{r}_{i}$, is given by

$$
\begin{aligned}
\sigma^{2}\left\{\frac{1}{n} \sum_{i=1}^{n} \overline{\mathbf{p}^{2}}\left(\mathbf{r}_{i}\right)\right\}= & \frac{1}{n} \sigma^{2}\left(\overline{\mathbf{p}^{2}}\right) \\
& +\frac{1}{n^{2}} \sum_{i=1}^{n} \sum_{j \neq 1}^{n} \operatorname{cov}\left\{\overline{\mathbf{p}^{2}}\left(\mathbf{r}_{i}\right), \overline{\mathbf{p}^{2}}\left(\mathbf{r}_{j}\right)\right\} .
\end{aligned}
$$

If the positions represent independent samples of the stochastic process (which means that they should be spaced at least by half a wavelength ${ }^{25,26}$ ) the second term on the right side of Eq. (5) vanishes, and the resulting variance is inversely proportional to the number of positions. It follows that the standard deviation will decrease with the inverse of the square root of $n$. Considering that the normalized variance of an exponential distribution is equal to 1 , it follows that it is necessary to average over a very large number of independent positions if a small variance of the spatial average is required.

\section{B. Frequency averaging}

In the same way as with the spatial averaging, the variance of the mean-square pressure over a number of adjacent frequencies will decrease with the inverse of the number of

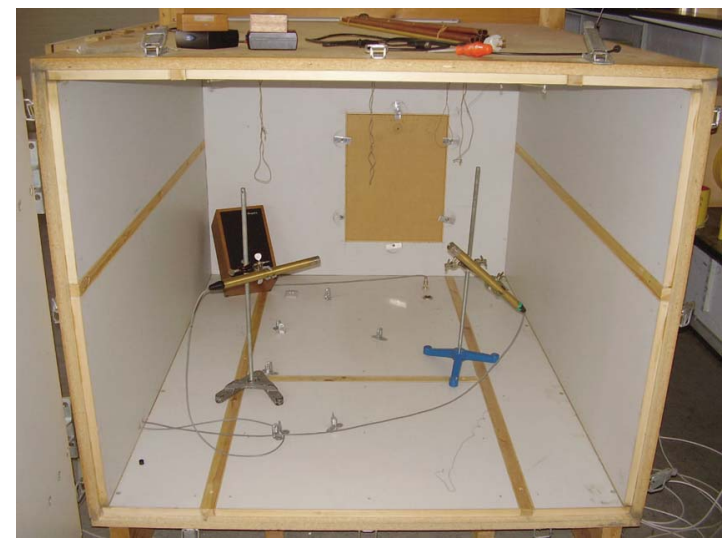

FIG. 1. (Color online) The reverberation chamber with the measurement rig. The loudspeaker is used for measuring the reverberation time.

independent samples. The frequency separation between independent samples depends on the losses of the room. The separation should satisfy ${ }^{23}$

$$
\Delta f>\frac{6}{T_{60}} .
$$

The mean-square pressure is also exponentially distributed when regarded as a function of the frequency. Therefore it is necessary to average over a large number of independent frequencies in order to obtain a small variance of the frequency average. With averaging over a wide band of width $B$ the variance is reduced by a factor of $B T_{60} \log e / 3$ $\approx B T_{60} / 6.9$. $^{19,27}$

\section{EXPERIMENTAL SETUP}

\section{A. Realizing the diffuse field}

If the measurements are carried out in a large reverberation room, the sound pressure level generated by the transmitter microphone will be low and it will be necessary to use very long microphone cables, which may have an adverse effect on the signal-to-noise ratio. Therefore, the measurements were carried out in a miniature reverberation room [a scale model (1:5) of the reverberation rooms at DTU]. The reverberation chamber has a volume of approximately $2 \mathrm{~m}^{3}$; see Fig. 1. The reverberation time of the room was measured in one-twelfth octave bands with a Brüel \& Kjær (B\&K) PULSE analyzer using the conventional interrupted noise method and averaging over repeated decays (five decays at five positions). The reverberation time and the associated spatial standard deviation are presented in Fig. 2. The Schroeder frequency of the room was estimated to be about $1 \mathrm{kHz}$. This is perfectly adequate for microphone calibration because the difference between the diffuse-field sensitivity and the pressure sensitivity of a microphone is very small below $1 \mathrm{kHz}$.

\section{B. Measurement system}

The measurement setup was composed of a $\mathrm{B} \& \mathrm{~K}$ reciprocity apparatus of type 5998, a microphone amplifier B\&K type NEXUS model 2690, and a B\&K PULSE multichannel analyzer. The receiver microphone was connected to a pre- 


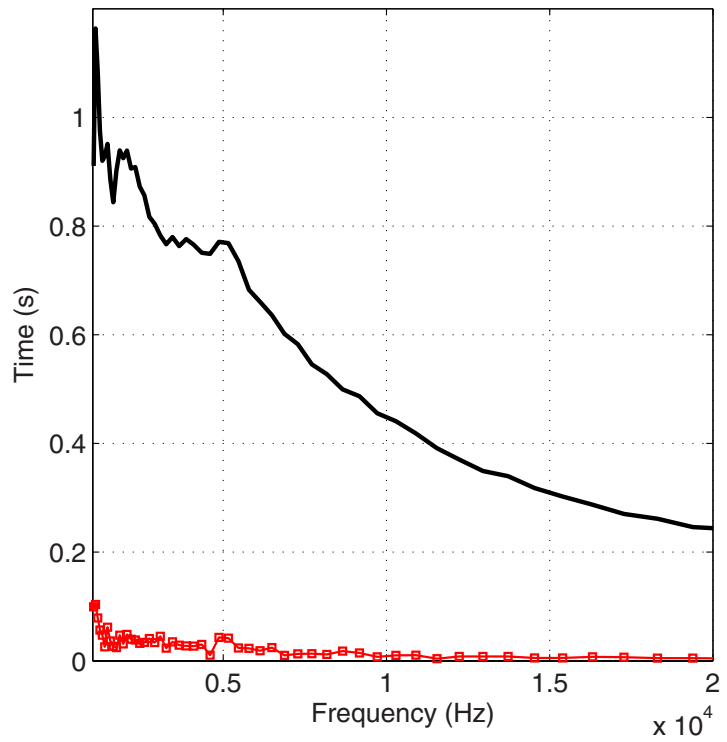

FIG. 2. (Color online) The reverberation time measured in the reverberation chamber. The thick solid line is the reverberation time and the thin solid line is the spatial standard deviation.

amplifier with a $20 \mathrm{~dB}$ built-in amplifier. The influence of the load of the preamplifier was eliminated using the conventional insert voltage technique. ${ }^{8}$ The preamplifiers were attached to cylindrical rods of the same diameter as the microphones and sufficiently long to approximate semi-infinite cylinders in the frequency range of concern. Figure 3 shows a block diagram of the measurement setup. The ratio of receiver-transmitter output voltages was measured with fast Fourier transform (FFT) analysis using synchronized pseu- dorandom noise. Determination of the impulse response requires a fine frequency sampling of the frequency response. Thus, it was necessary to measure the frequency response in frequency steps of $\Delta f=0.5 \mathrm{~Hz}$. In order to cover the entire frequency range with such fine resolution sequential "zoom FFT" measurements were carried out. Each zoom had a bandwidth of $3.2 \mathrm{kHz}$ and 6400 frequency lines.

\section{Separation of the reverberant response}

The separation of the direct and reverberant response can be carried out using a time-selective procedure very similar to the one described in Ref. 10. The procedure applied in this case was as follows: (a) The transfer function between the signals of two microphones was measured; (b) at low and high frequencies this frequency response was taken smoothly to zero by applying a bandpass filter; (c) an inverse discrete Fourier transform was calculated; (d) a timeselective window was applied to the resulting impulse response in order to separate the direct and the reverberant response; and (e) a discrete Fourier transform was applied to the reverberant part of the impulse response. The transfer function in (a) was measured using the procedure and parameters described in the previous subsection. The bandpass filter used in (b) had roll-off frequencies of 2 and $23 \mathrm{kHz}$. The time-selective window used in (d) was a Tukey window with a length of $1.99 \mathrm{~s}$, and the percentage of the smoothing portion at the ends of the window was of $0.1 \%$ of the length of the window. The Tukey window was selected because it does not modify the impulse response beyond the smoothing at

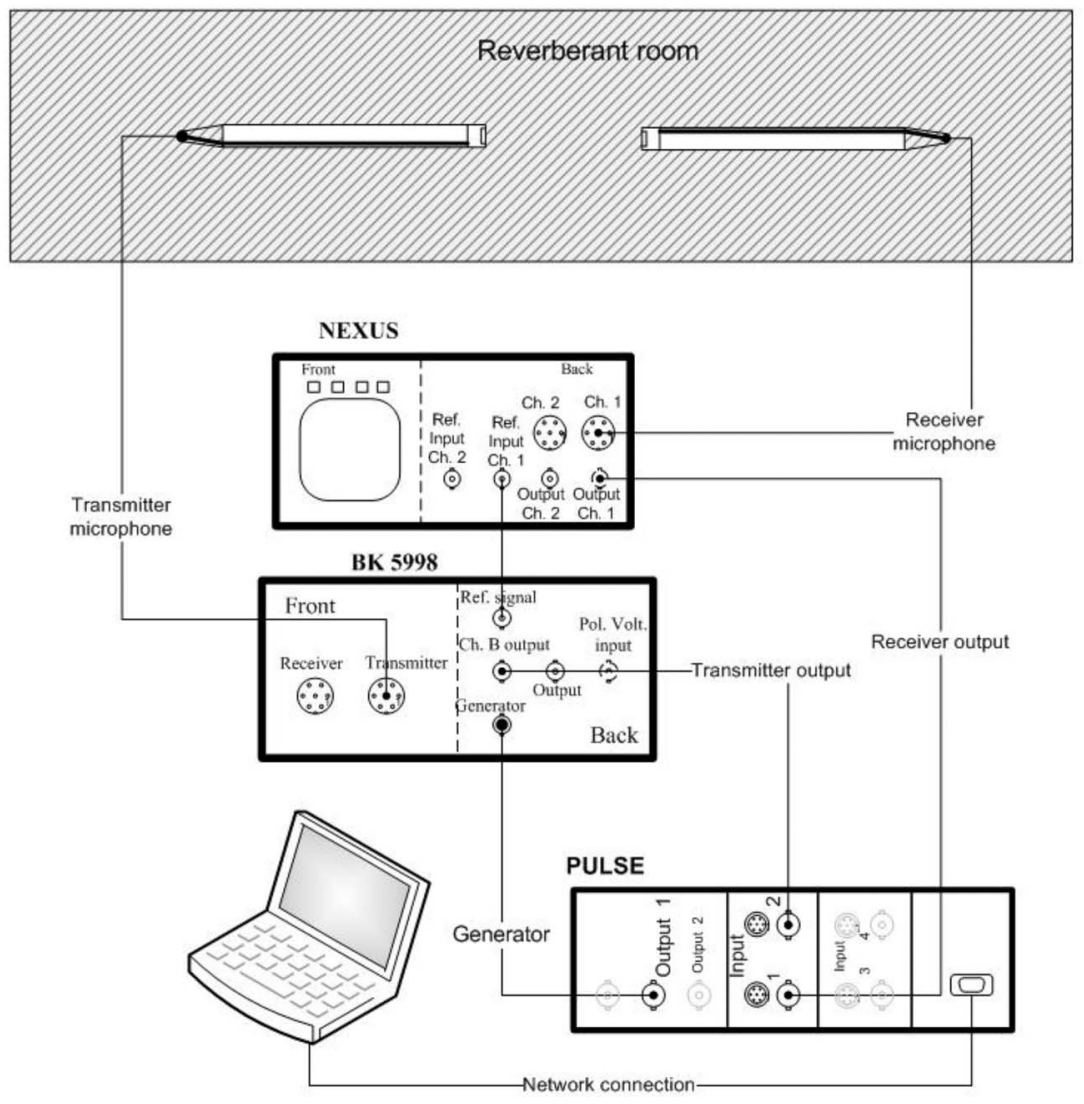

FIG. 3. Block diagram of the measurement system. 
(a) Face to face at $24 \mathrm{~cm}$

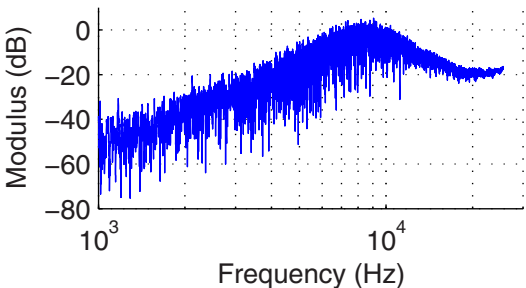

(c)

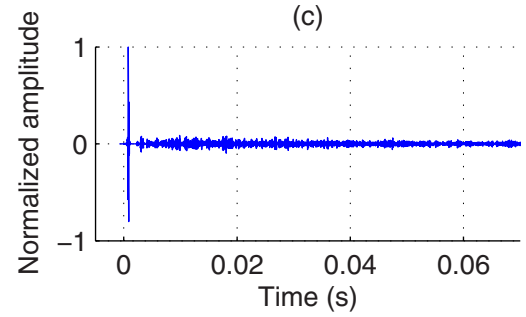

(e)

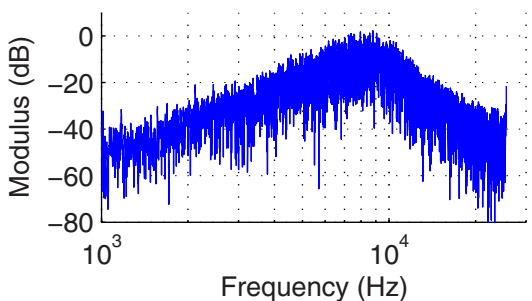

(b) Arbitrarily oriented at $60 \mathrm{~cm}$

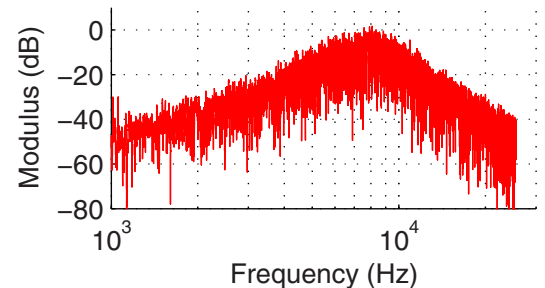

(d)

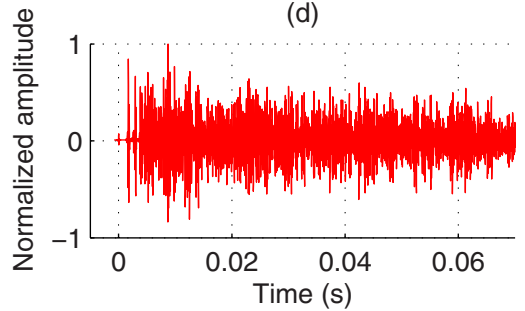

(f)

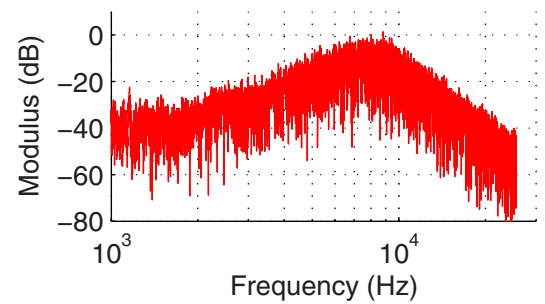

FIG. 4. (Color online) Frequency and impulse responses measured with different configurations in the reverberation chamber. Graphs in the left column display data obtained from measurements of the transfer function with the microphones face to face at a distance of $24 \mathrm{~cm}$ from each other; graphs in the right column display data obtained from measurements of the transfer function with the microphones oriented at arbitrary directions and at approximately $60 \mathrm{~cm}$ from each other. Graphs (a) and (b) show the measured ratios of voltages, graphs (c) and (d) are the impulse responses, and graphs (e) and (f) are the reverberant ratios of voltages. the ends of the window; the window starts at $0.002 \mathrm{~s}$. In this way, the reverberant impulse response was separated from the direct response.

\section{EXPERIMENTAL RESULTS}

Figure 4 shows the transfer impedance between two 1 in. laboratory standard (LS1) microphones measured at two different sets of positions in the reverberant room: in one case the microphones were face to face at a distance of $24 \mathrm{~cm}$ from each other, and in the other case the microphones had arbitrary orientations and were placed at a distance of approximately $60 \mathrm{~cm}$ from each other.

The fluctuations of the frequency response shown in Fig. 4(a) start to decrease significantly above $10 \mathrm{kHz}$ when the microphones face each other at a short distance. This is a consequence of the high ratio of the direct field to the reverberant field in this frequency range, and this can be expected because LS1 microphones are highly directional. By contrast the fluctuations of the transfer impedance between the microphones oriented at arbitrary directions do not decrease at high frequencies. The impulse responses shown in Figs. 4(c) and 4(d) confirm this interpretation; in Fig. 4(c) the direct impulse response is much larger than the reverberant response whereas in Fig. 4(d) the two responses are of similar amplitude. Finally, the separated reverberant responses shown in Figs. 4(e) and 4(f) were determined using the procedure described above. As can be seen, the reverberant responses are very similar for the two cases under consideration. This indicates that it is possible to measure at any position in the reverberation chamber and to separate the reverberant response from the direct sound or early reflections, leaving only the reverberant part to be used for further analysis.
The next step is to calculate the diffuse-field sensitivity from the reverberant response. Figure 5 shows the diffusefield sensitivity of an LS1 microphone. The calculated sensitivity, which complies with the definition in Ref. 16, fluctuates dramatically with the frequency. These fluctuations are a consequence of the realization of the sound field and not a property of the microphone itself. Thus, in order to reduce the fluctuations to an acceptable level the sensitivity must be averaged over different positions in the chamber and over frequency. It was also mentioned in Sec. II that the reciprocity factor is only valid on average. According to Eq. (5) one would have to average over about 1850 independent positions in the reverberation chamber in order to reduce the

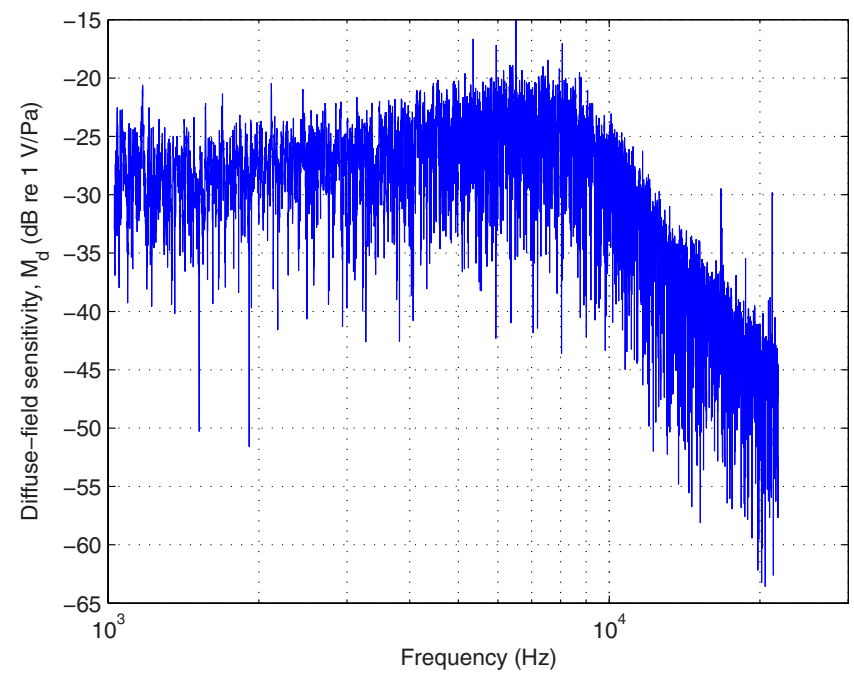

FIG. 5. (Color online) Diffuse-field sensitivity of an LS1 microphone calculated from the reverberant transfer function for a single microphone configuration using the reciprocity technique. 
(a)

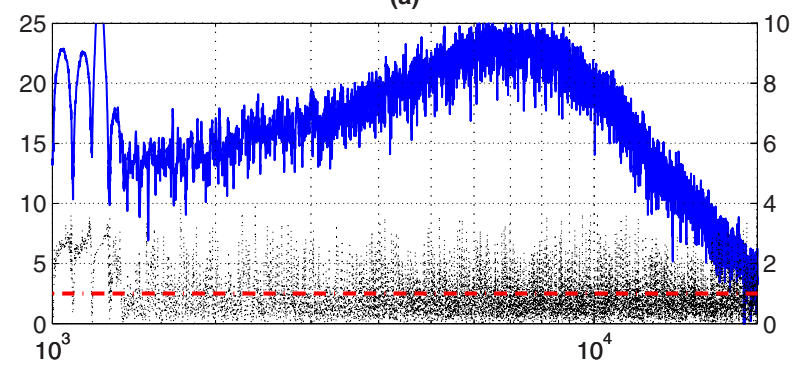

(b)

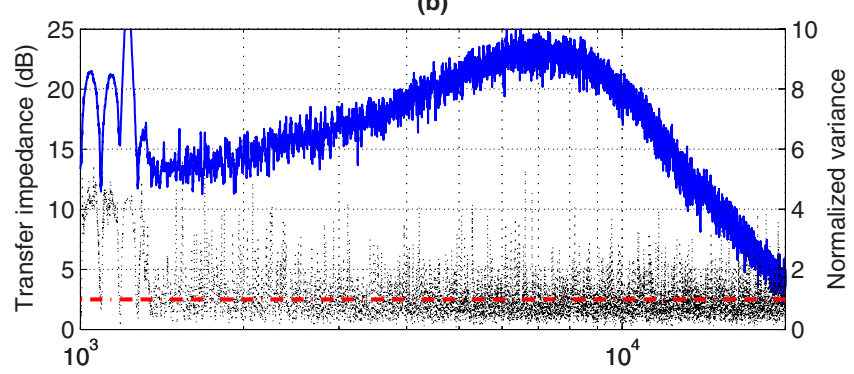

(c)

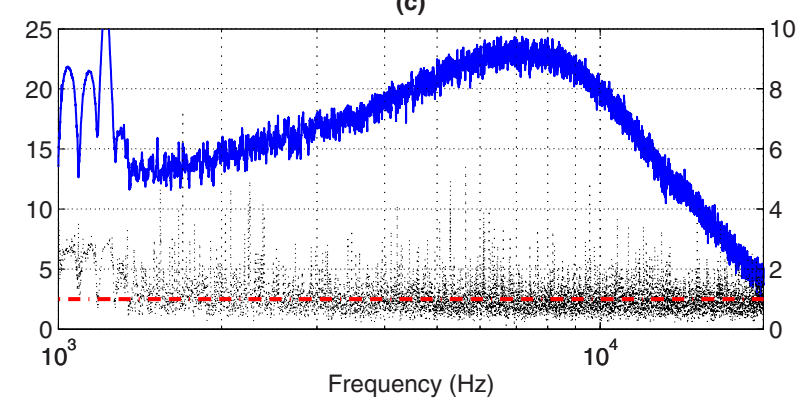

FIG. 6. (Color online) Spatial average and normalized variance of the transfer impedance between two LS1 microphones determined from measurements in the reverberation chamber at (a) 4 different positions, (b) 9 different positions, and (c) 16 different positions. The thick solid line is the modulus of the transfer impedance (in decibels), the thin dotted line is the normalized variance, and the thick dash-dotted line is the predicted normalized variance.

standard deviation of the mean-square sensitivity to $0.1 \mathrm{~dB}$. This is prohibitive, not only from a practical point of view but also because there simply are not so many independent positions in the chamber. This leads to the conclusion that frequency averaging is also needed.

Before proceeding, it should be decided whether the averaging should be made before or after the calculation of the sensitivity. Averaging after the sensitivity is calculated (as in Fig. 5) requires that the fine structure of the transfer impedance is exactly the same for the three microphone combinations; thus the ratio $R=Z_{i j} Z_{i k} / Z_{j k}$ should be similar to the three transfer impedances. However, this may not always be the case; differences between the transfer impedances' "fine structure" may occur because of temperature changes and small random positioning errors that cannot easily be avoided when manual positioning is used. A more robust approach is to do the averaging over frequency of each squared transfer impedance before the ratio $R$ is calculated. This is the approach adopted here.

Figure 6 shows the spatial average and the normalized variance of the squared modulus of the transfer impedance

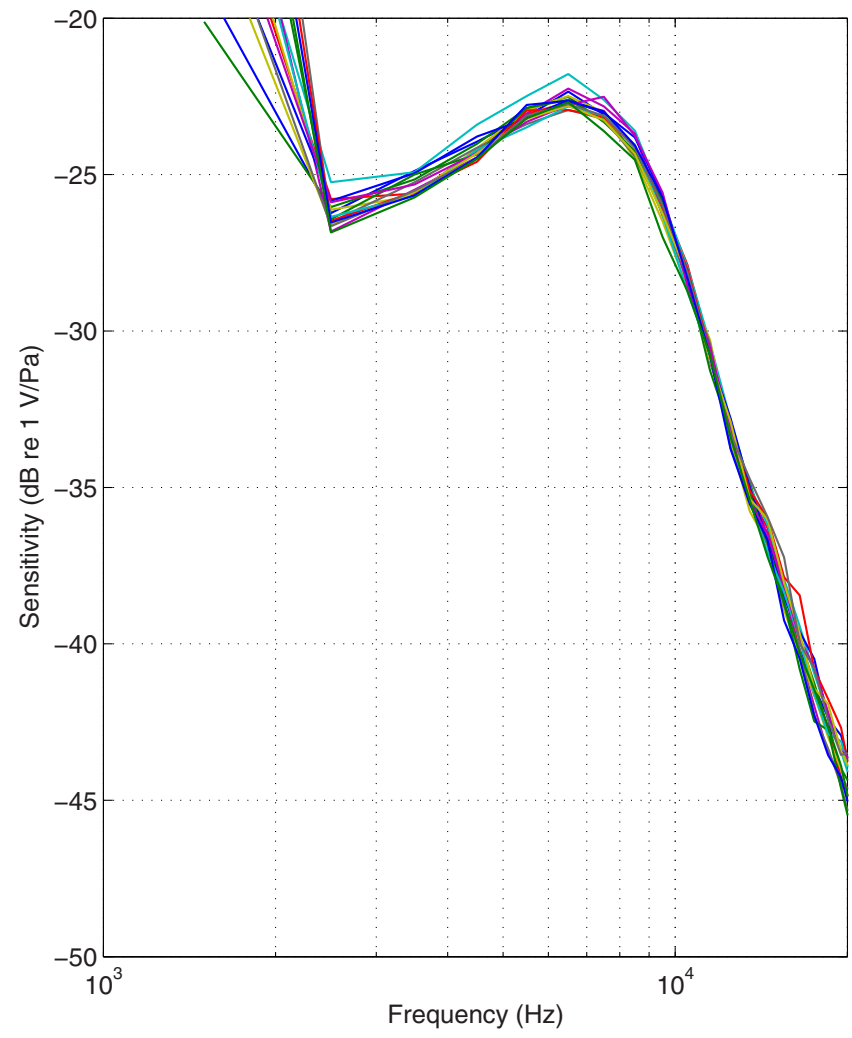

FIG. 7. (Color online) Frequency averaged diffuse-field sensitivity of an LS1 microphone determined from measurements at 16 different configurations in the scale model of a reverberation room.

between microphones 1 and 2, determined from measurements at 4, 9, and 16 different positions in the reverberation chamber. Although the fluctuations decrease visibly when the number of averages increases, it is still clear that a much larger number of measurements at independent positions in the chamber would be required if a smoother realization of the final diffuse-field sensitivity is required. The value of the normalized variance oscillates around 1 . This is consistent with the assumption that the squared transfer impedance is proportional to the mean-square pressure and therefore has an exponential distribution.

Figure 7 shows the results of averaging the diffuse-field sensitivity of an LS1 microphone measured at 16 random pairs of positions in the reverberation room over frequency. For each configuration the three squared transfer impedances are averaged over frequency bands with a width of $1 \mathrm{kHz}$, as described above. It can be seen that the fluctuations disappear almost completely. However, there are still differences between the sensitivities obtained from different microphone positions in the reverberation room. In addition to the uncertainty due to the variation with position of the sound power output of the transmitter microphone, ${ }^{20,21}$ these differences might be caused, e.g., by changes of the environmental conditions during the measurements. However, the most important factor is undoubtedly the variability of the sound field itself. The spatial averaging will reduce the standard deviation by a factor of $\sqrt{n}$ [see Eq. (5)], and the frequency averaging will reduce it in inverse proportion to $\sqrt{B T_{60}}$, as mentioned above. 


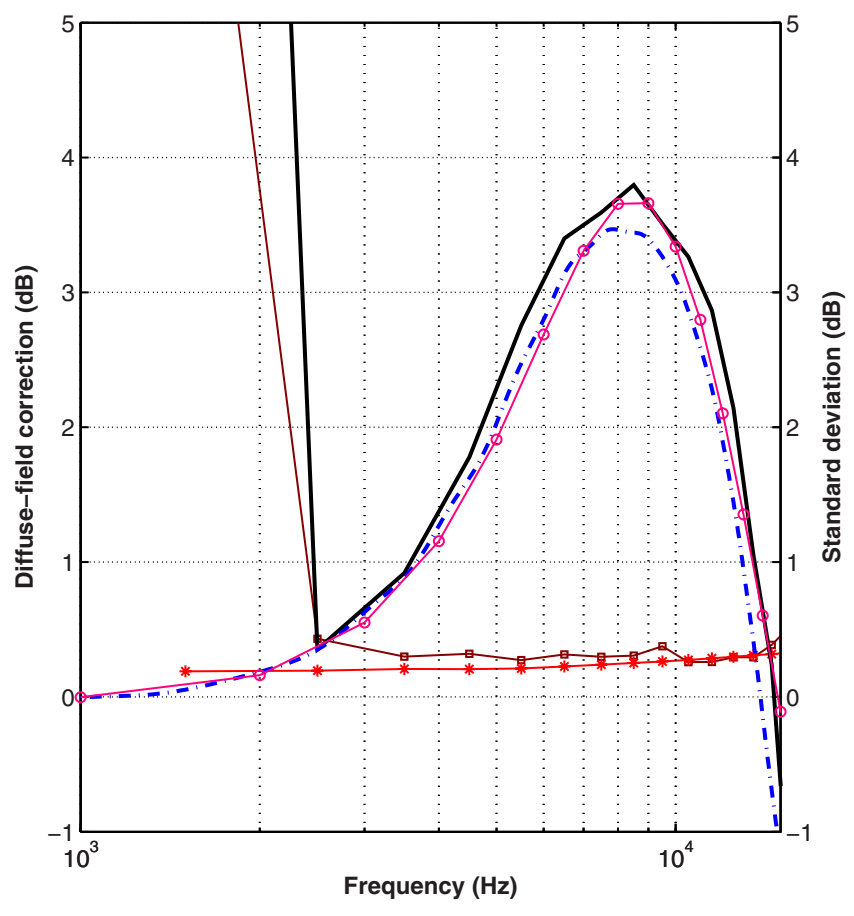

FIG. 8. (Color online) Diffuse-field correction of LS1 microphones. The thick solid line is the average diffuse-field correction, the dash-dotted line is the random-incidence correction from Ref. 15, the line with circular markers is the random-incidence correction calculated using the boundary element method, the line with square markers is the measured spatial standard deviation of the diffuse-field correction, and the line with star markers is the predicted spatial standard deviation of the diffuse-field sensitivity.

Finally, Figs. 8 and 9 show the average diffuse-field correction of three LS1 microphones and three LS2 (0.5 in.) microphones, respectively. The correction has been determined from the diffuse-field sensitivity averaged both over frequency and 16 sets of microphone positions in the reverberation chamber. The estimate of the diffuse-field correction is compared with estimates of the random-incidence correction from Ref. 15 and a numerical calculation determined using the boundary element method (BEM). The velocity distributions of the membranes of LS1 and LS2 microphones used in the BEM calculations were measured using a laser vibrometer.

One cannot trust the diffuse-field results for LS1 microphones below $3 \mathrm{kHz}$; this is due to the time-selective procedure applied for separating the free-field and diffuse responses, and more specifically because of the roll-off frequencies of the passband filter used in the time-selective procedure. This is a step that could easily be improved by designing a filter with different roll-off frequencies. Above the lower roll-off frequency the agreement between the three estimates is within $0.1 \mathrm{~dB}$ below the resonance frequency (about $8 \mathrm{kHz}$ for LS1 microphones and $18 \mathrm{kHz}$ for LS2 microphones). Around and above the resonance frequency the agreement is still reasonably good, about $0.3 \mathrm{~dB}$, but some systematic deviations occur, especially in the case of LS2 microphones. The BEM calculations agree slightly better with the diffuse-field measurements than with the randomincidence measurements. Introducing small, systematic errors in the angle of incidence in the BEM calculations indicates that the random-incidence sensitivities at high

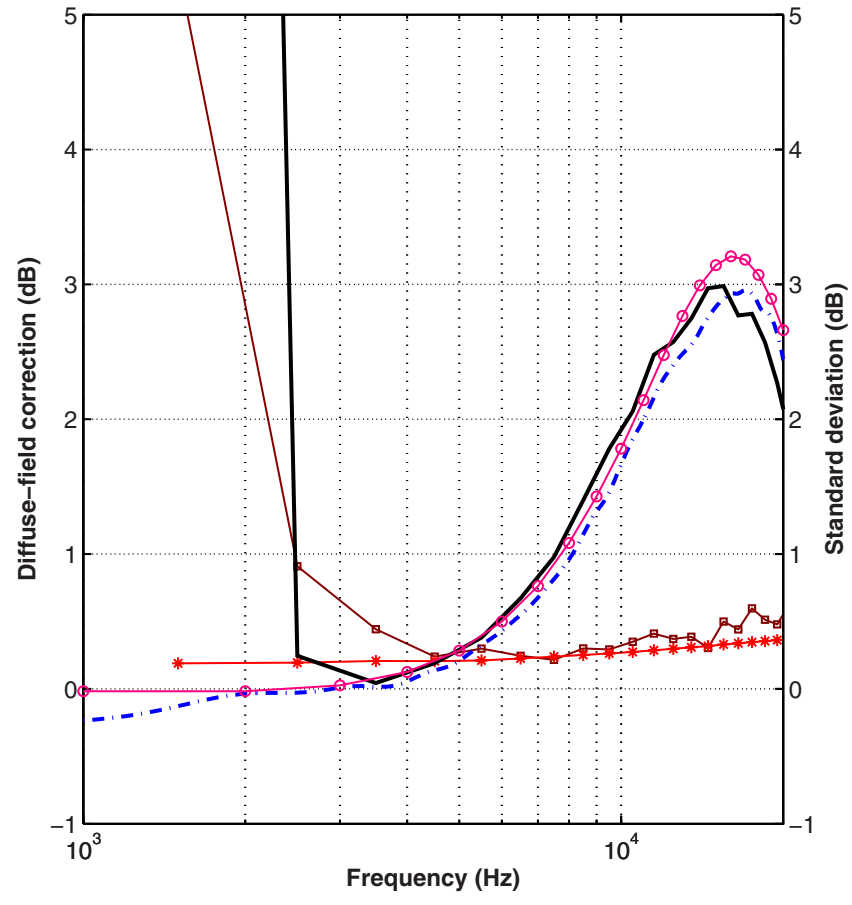

FIG. 9. (Color online) Diffuse-field correction of LS2 microphones. The thick solid line is the average diffuse-field correction, the dash-dotted line is the random-incidence correction from Ref. 15, the line with circular markers is the random-incidence correction calculated using the boundary element method, the line with square markers is the measured spatial standard deviation of the diffuse-field correction, and the line with star markers is the predicted spatial standard deviation of the diffuse-field sensitivity.

frequencies may have been underestimated because of a systematic error in the incidence angle in the anechoic room, and this is a likely explanation for the observed deviations. Nevertheless, the overall agreement is fair, and the good agreement confirms that the diffuse-field and the randomincidence corrections are equivalent when the definition of the diffuse-field sensitivity in Ref. 16 is interpreted as an ensemble average of realizations of reverberant interference fields.

Figures 8 and 9 also show the spatial standard deviations of the diffuse-field sensitivities (in decibels) and values predicted from the expression

$$
\sigma\left\{20 \log \left(M_{d}\right)\right\} \approx 5 \log e \sqrt{\frac{3}{B T_{60} \log e}} \approx \frac{5.7}{\sqrt{B T_{60}}} .
$$

Equation (7) follows from an expression derived by Lubman $^{19}$ and Schroeder ${ }^{27}$ for the spatial variance of the mean-square pressure averaged over a frequency band combined with the fact that the diffuse-field sensitivity is proportional to the fourth root of the frequency averaged meansquare pressure; cf. Eq. (3). The measured standard deviations are in good agreement with the predictions.

\section{CONCLUSIONS}

The definition of the diffuse-field sensitivity given in Ref. 16 leads to dramatic fluctuations with the frequency and must be combined with spatial averaging and frequency smoothing. The diffuse-field sensitivities of LS1 and LS2 microphones have been determined in a small reverberation 
chamber using the reciprocity technique. The experimental procedure requires (i) measurement of the electrical transfer impedance between two microphones, (ii) a time-selective procedure to separate the direct sound field from the reverberant sound field, and (iii) averaging of the resulting diffuse-field sensitivity both spatially and in the frequency domain. The resulting diffuse-field sensitivity has been found to agree satisfactorily with the random-incidence sensitivity and with the results of BEM calculations based on measured membrane velocity distributions.

The main advantage of determining the diffuse-field sensitivity using reciprocity compared with determining the random-incidence correction is that the former provides a direct, absolute estimate whereas the random-incidence correction is determined from a relative measurement. It is also worth mentioning that measurement of the random-incidence sensitivity requires facilities that are not always available, such as an anechoic room, and such measurements can be very time consuming if a high accuracy is wanted.

\section{ACKNOWLEDGMENTS}

This project has been carried out with support from the Danish Research Council for Technology and Production Sciences (FTP).

${ }^{1}$ F. Jacobsen and T. Roisin, "The coherence of reverberant sound fields," J. Acoust. Soc. Am. 108, 204-210 (2000).

${ }^{2}$ L. L. Beranek, Acoustical Measurements (AIP/ASA, New York, 1988).

${ }^{3}$ Microphone Handbook (Brüel \& Kjær, Nærum, Denmark, 1996).

${ }^{4}$ W. R. MacLean, "Absolute measurement of sound without a primary standard," J. Acoust. Soc. Am. 12, 140-146 (1940).

${ }^{5}$ R. K. Cook, "Absolute pressure calibration of microphones," J. Acoust. Soc. Am. 12, 415-420 (1941).

${ }^{6}$ A. L. DiMattia and F. M. Wiener, "On the absolute pressure calibration of condenser microphones by the reciprocity method," J. Acoust. Soc. Am. 18, 341-344 (1946).

${ }^{7} \mathrm{~W}$. Wathen-Dunn, "On the reciprocity free-field calibration of microphones," J. Acoust. Soc. Am. 21, 542-546 (1949).

${ }^{8}$ IEC International Standard 61094-2, Measurement microphones Part 2: Primary method for pressure calibration of laboratory standard micro- phones by the reciprocity technique (1995).

${ }^{9}$ IEC International Standard 61094-3, Measurement microphone Part 3: Primary method for free-field calibration of laboratory standard microphones by the reciprocity technique (1995).

${ }^{10}$ S. Barrera-Figueroa, K. Rasmussen, and F. Jacobsen, "A time-selective technique for free-field reciprocity calibration of condenser microphones," J. Acoust. Soc. Am. 114, 1467-1476 (2003).

${ }^{11}$ H. G. Diestel, "Reciprocity calibration of microphones in a diffuse sound field," J. Acoust. Soc. Am. 33, 514-518 (1961).

${ }^{12}$ T. Nakajima, "Reciprocity calibration of laboratory standard microphones in a diffuse sound field," Researches of the Electrotechnical Laboratory 76, 1-87 (1970). (in Japanese.)

${ }^{13} \mathrm{M}$. Vorländer and H. Bietz, "Novel broad-band reciprocity technique for simultaneous free-field and diffuse-field microphone calibration," Acustica 80, 365-377 (1994).

${ }^{14}$ IEC International Standard 61183, Random incidence and diffuse-field calibration of sound level meters (1994).

${ }^{15}$ S. Barrera-Figueroa, K. Rasmussen, and F. Jacobsen, "On experimental determination of the random-incidence response," J. Acoust. Soc. Am. 121, 2628-2636 (2007).

${ }^{16}$ IEC International Standard 61094-1, Measurement microphones Part 1: Specifications for laboratory standard microphones (1992).

${ }^{17} \mathrm{H}$. G. Diestel, "Probability distribution of sinusoidal sound pressure in a room," J. Acoust. Soc. Am. 35, 2019-2022 (1963).

${ }^{18}$ R. V. Waterhouse, "Statistical properties of reverberant sound fields," J. Acoust. Soc. Am. 43, 1436-1444 (1968).

${ }^{19}$ D. Lubman, "Fluctuations of sound with position in a reverberant room," J. Acoust. Soc. Am. 44, 1491-1502 (1968).

${ }^{20}$ F. Jacobsen, "The diffuse sound field," Report No. 27, The Acoustics Laboratory, Technical University of Denmark, 1979.

${ }^{21}$ A. D. Pierce, Acoustics: An Introduction to Its Physical Principles and Applications (AIP/ASA, New York, 1989).

${ }^{22}$ M. R. Schroeder, "Die statistischen parameter der frequenzkurven von grossen räumen (statistical parameters of frequency responses in large rooms)," Acustica 4, 594-600 (1954).

${ }^{23}$ M. R. Schroeder, "Frequency-correlation of frequency responses in rooms," J. Acoust. Soc. Am. 34, 1819-1823 (1963).

${ }^{24}$ S. Müller and P. Massarani, "Transfer-function measurements with sweeps," J. Audio Eng. Soc. 49, 443-471 (2001).

${ }^{25}$ R. K. Cook, R. V. Waterhouse, R. D. Berendt, S. Edelman, and M. C. Thompson, Jr., "Measurement of correlation coefficients in reverberant sound fields," J. Acoust. Soc. Am. 27, 1072-1077 (1955).

${ }^{26}$ D. Lubman, "Spatial averaging in a diffuse sound field," J. Acoust. Soc. Am. 46, 532-534 (1969).

${ }^{27}$ M. R. Schroeder, "Effect of frequency and space averaging on the transmission response of multimode media," J. Acoust. Soc. Am. 46, 277-283 (1969). 